\title{
Beneficial effects of short-term nutritional counselling at the primary health-care level among Brazilian adults
}

\author{
Daniela Saes Sartorelli ${ }^{1}$, Elaine Cristina Sciarra ${ }^{1}$, Laércio Joel Franco ${ }^{2}$ and \\ Marly Augusto Cardoso ${ }^{1, *}$ \\ 'Department of Nutrition, School of Public Health, University of São Paulo (USP), Av. Dr Arnaldo 715, São Paulo, \\ SP 01246-904, Brazil: ${ }^{2}$ Department of Social Medicine, School of Medicine of Ribeirão Preto, University of \\ São Paulo (USP), Av. Bandeirantes 3900, Ribeirão Preto, SP 14049-900, Brazil
}

Submitted 29 September 2004: Accepted 21 March 2005

\begin{abstract}
Objective: To evaluate the impact of a low-cost nutritional intervention in changing the lifestyle of adults.

Design: Randomised clinical trial.

Setting: Primary health-care centre in São José do Rio Preto, São Paulo State, Brazil. Subjects: We randomly assigned 104 adults (83 women and 21 men aged 30-65 years, body mass index $24-35 \mathrm{~kg} \mathrm{~m}^{-2}$, non-diabetic) into two groups: nutrition counselling and control. Each subject in the intervention group received three individualised nutritional counselling sessions during the first 6 months aimed at increasing intakes of fruits, vegetables and olive oil, reducing saturated fat and improving physical activity. Body composition, biochemical indicators and lifestyle were assessed at baseline and at 6 months and 1 year in both groups.

Results: After 6 months of follow-up, body weight, waist circumference, diastolic blood pressure, fasting blood glucose, total and low-density lipoprotein cholesterol, total and saturated fat, and dietary energy and cholesterol levels showed a more significant decrease among subjects in the intervention group than in the control group $(P<0.05)$. Moreover, the intervention group showed significantly greater improvement in each intervention goal, such as reduced intake of saturated fat and increased intakes of fruits, vegetables, fibre and olive oil $(P<0.05)$. After 12 months of follow-up, most of the outcomes were maintained.

Conclusions: The low-cost nutritional intervention programme improved serum lipids profile and weight control, and appeared to be feasible for use at a primary healthcare centre in a developing country.
\end{abstract}

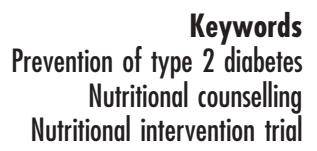

The burden of chronic diseases, especially type 2 diabetes and cardiovascular diseases, is rapidly increasing worldwide. In developing countries, increased rates have been estimated to occur at younger ages, representing an important public health problem ${ }^{1}$. As is the case for many developing countries, the joint effects of changes in lifestyle and ageing of the population are the main risk factors for type 2 diabetes and other chronic diseases in the Brazilian population ${ }^{2}$.

The Diabetes Prevention Program (DPP) ${ }^{3}$ and The Finnish Diabetes Prevention Study (DPS) ${ }^{4}$ have reported convincing evidence that type 2 diabetes can be prevented by the adoption of a healthy lifestyle in overweight subjects with impaired glucose tolerance. Although intensive lifestyle intervention can result in significant improvements in a range of clinical and metabolic variables, it is uncertain whether nutritional intervention programmes that require fewer resources than those provided in the DPP or DPS, being feasible in the health-care systems of developing countries, are effective in achieving weight reduction, increased physical activity and improved quality of life $\mathrm{e}^{5}$.

We carried out a randomised controlled trial to investigate the effect of a 6-month primary-care intervention in nondiabetic adults. In the current study we tested the hypothesis that low-cost individualised nutritional counselling addressing the primary prevention of type 2 diabetes and other chronic diseases would: (1) improve weight control and serum lipids profile; (2) improve patterns of food intake and physical activity; and (3) be feasible at a primary health-care unit in a developing country.

\section{Research design and methods}

\section{Screening and recruitment}

Study subjects were recruited primarily through the screening of members of high-risk groups, such as 
overweight/obese adults or first-degree relatives of patients with type 2 diabetes. Advertisements were also used to recruit the study subjects. Eligibility criteria included age of 30-65 years, body mass index (BMI) of $24-35 \mathrm{~kg} \mathrm{~m}^{-2}$, and non-diabetic status based on 1998 World Health Organization (WHO) criteria ${ }^{6}$. The exclusion criteria included a prior diagnosis of type 2 diabetes, cardiovascular, kidney, liver, thyroid or renal diseases, endocrine disorders, cancer or AIDS; use of medications that may influence lipid or carbohydrate metabolism (thiazide diuretics, beta-blockers, glucocorticoids, weightloss medications, thyroid hormone or hypolipidaemic medications); pregnancy or childbearing; weight loss $>10 \%$ in the past 6 months; systolic blood pressure $>180 \mathrm{mmHg}$ or diastolic blood pressure $>105 \mathrm{mmHg}$; excessive alcohol intake; fasting plasma triglycerides $>600 \mathrm{mg} \mathrm{dl}^{-1}$; close contact with individuals included in the study; and illiteracy.

\section{Study protocol}

Of the 259 volunteers (203 women, 56 men) contacted from April 2000 to March 2001, 104 eligible subjects were randomly assigned with stratification by sex and glucose tolerance status. A number was assigned to each participant. Then, numbers were selected at random to one of the two treatment groups. Excluded individuals with BMI $>35 \mathrm{~kg} \mathrm{~m}^{-2}$, diabetes or other diseases were assigned to specific medical assistance. All participants were blinded to treatment assignment for the duration of the study. However, the staff members involved in the intervention had to be aware of the group assignment. The protocol was reviewed and approved by the Ethics Committee of the School of Medicine of São José do Rio Preto and written informed consent was obtained from each participant.

At baseline, a health check was performed by the staff research members, and all participants received written information and a $30 \mathrm{~min}$ group session addressing the importance of a healthy lifestyle, which is the usual primary care given to overweight adults.

Subjects in the control group were scheduled for further health checks at 6 months and 1 year after baseline. Participants in the control group did not receive any individualised intervention during follow-up but received the same health check and blood sampling as the intervention group. At the end of the study, individuals in the control group started to receive individualised nutritional counselling.

Subjects in the intervention group were scheduled for three individualised dietary counselling sessions during the first 6 months of intensive lifestyle intervention and further health checks at 6 months and 1 year from baseline. The intervention group received a diet prescription provided by a nutritionist with a food exchange list and was encouraged to practise at least $30 \mathrm{~min}$ of walking per day. The dietary interventions included increased intakes of olive oil, fruits (at least 2 servings day ${ }^{-1}$ ), vegetables (at least 5 servings day $^{-1}$ ) and skimmed dairy products ( 2 or 3 servings day ${ }^{-1}$ ), together with reduced intake of saturated fat $(<10 \%$ of energy by reducing red meat - less than 2 servings day ${ }^{-1}$ ) and keeping the consumption of total fat around 30\% of energy without emphasis on total energy restriction. The subjects were encouraged to reduce their consumption of sweets and fried foods. After the second health check, 6 months from baseline, the subjects did not receive any further intervention.

The sample size (at least 50 subjects in the intervention group) was based on detecting an improvement in serum lipid profile (at least $7-10 \%$ reduction in total cholesterol) at $80 \%$ power with a two-tailed level of significance of $5 \%$.

\section{Assessments}

During the health checks, anthropometric measures, biochemical indicators, lifestyle (dietary intake and physical activity) and demographic variables were assessed at baseline and after 6 months and 1 year. Age, gender and other demographic variables were obtained using a structured questionnaire. Food consumption was assessed using a food-frequency questionnaire previously validated and adapted to the present study ${ }^{7,8}$. Subjects were asked by a trained nutritionist about frequency of consumption of foods (75 items) and portion sizes during the previous month. The nutrient composition of the diets was determined using the Dietsys software ${ }^{9}$ version 4.01. Physical activity was assessed by a structured physical activity questionnaire previously evaluated for reproducibility (Spearman coefficients ranging from 0.51 to 0.82 , unpublished data). Subjects were asked about frequency and time spent in practices of exercises, sports, leisure-time physical activities, usual work, walking and sedentary activities (such as watching television) during the previous week.

Body weight was measured with subjects wearing light clothing and no shoes using calibrated electronic scales (Plenna, model MEA-07400). Height was measured with a portable stadiometer. Waist circumference was measured at the level of the umbilicus. The average of three measurements of blood pressure using an automatic device (Omron model HEM-712 C; Omron Health Care, USA) with subjects in the sitting position, after a $10 \mathrm{~min}$ rest, was used to express systolic and diastolic pressures.

Fasting capillary blood glucose was evaluated using glucose oxidase strips (Glucometer Elite; Bayer). Individuals with fasting capillary glucose less than $200 \mathrm{mg} \mathrm{dl}^{-1}$ underwent a $75 \mathrm{~g}$ glucose tolerance test. Fasting and $2 \mathrm{~h}$ plasma glucose, and fasting serum levels of total cholesterol, lipoprotein fractions and triglycerides, were measured enzymatically. Glucose tolerance status was based on 1998 WHO criteria ${ }^{6}$.

\section{Statistical analysis}

We undertook an intention-to-treat analysis. Two-sided $t$-tests and chi-square tests were used to analyse differences between groups at baseline and during 
follow-up. Mean differences in outcomes between intervention and control groups are shown, with the 95\% confidence interval (CI). All statistical analyses were carried out using SPSS (version 10.0; SPSS Inc., Woking, Surrey, UK), with the level of significance set at $P<0.05$.

\section{Role of the funding source}

The sponsors of the study had no role in study design, data collection, data analysis, data interpretation or writing of the report.

\section{Results}

A total of 104 subjects (83 women and 21 men, 30-63 years old) were eligible and completed baseline examinations. Of these, 80 subjects attended the second examination (6 months after baseline) and 71 subjects attended the third examination (1 year after baseline), as shown in Fig. 1. At the second evaluation, three participants from the control group were excluded because they were taking medications that influence lipid or glucose metabolism. There were no statistical differences between participants who attended all the evaluations and those lost to follow-up with regard to $\mathrm{BMI}$, age, gender, income or glucose tolerance status. The drop-out rates, 20\% for the second and 30\% for the third evaluation, were similar to those reported in a previous study ${ }^{10}$ and were related to work commitments.
At baseline, characteristics and dietary intake of energy and macronutrients were similar between groups (Table 1). Family history of diabetes was reported by 52 participants (28/24 in the intervention/control group, respectively). The number of participants according to glucose tolerance status for intervention/control groups was $42 / 43$ normal, 3 / 3 impaired fasting glucose, $6 / 7$ impaired glucose tolerance. During the first 6 months of lifestyle counselling, the mean (\% of baseline body weight) of weight reduction was 3.1 and of weight gain was 0.4 in the intervention and control groups, respectively. Significantly greater improvements were seen at the 6-month follow-up in waist circumference $(-2.0 \%$ vs. $+0.2 \%)$, total cholesterol $(-12.3 \%$ vs. $-0.2 \%)$, low-density lipoprotein (LDL) cholesterol $(-15.5 \%$ vs. $+4.0 \%)$ and fasting plasma glucose $(-5.6 \%$ vs. $+0.1 \%)$ in the intervention group compared with the control group $(P<0.05)$. At 12 -month follow-up, when we evaluated maintenance of the changes in anthropometric measures and serum lipids profile, the differences in weight $(-3.1 \%$ vs. $+0.5 \%)$ and waist circumference $(-2.0 \%$ vs. $+0.1 \%)$ remained greater in the intervention group $(P<0.01)$. At this time, the reduction in LDL cholesterol levels $(-13.3 \%)$ in the intervention group was significant when compared with baseline data, but not different from the control group. Both groups had reduced serum total cholesterol after a year of follow-up. At 12-month follow-up we observed an increase in diastolic blood pressure of $4.5 \%$ in the control group (Table 2). After 6 and 12 months of follow-up, there

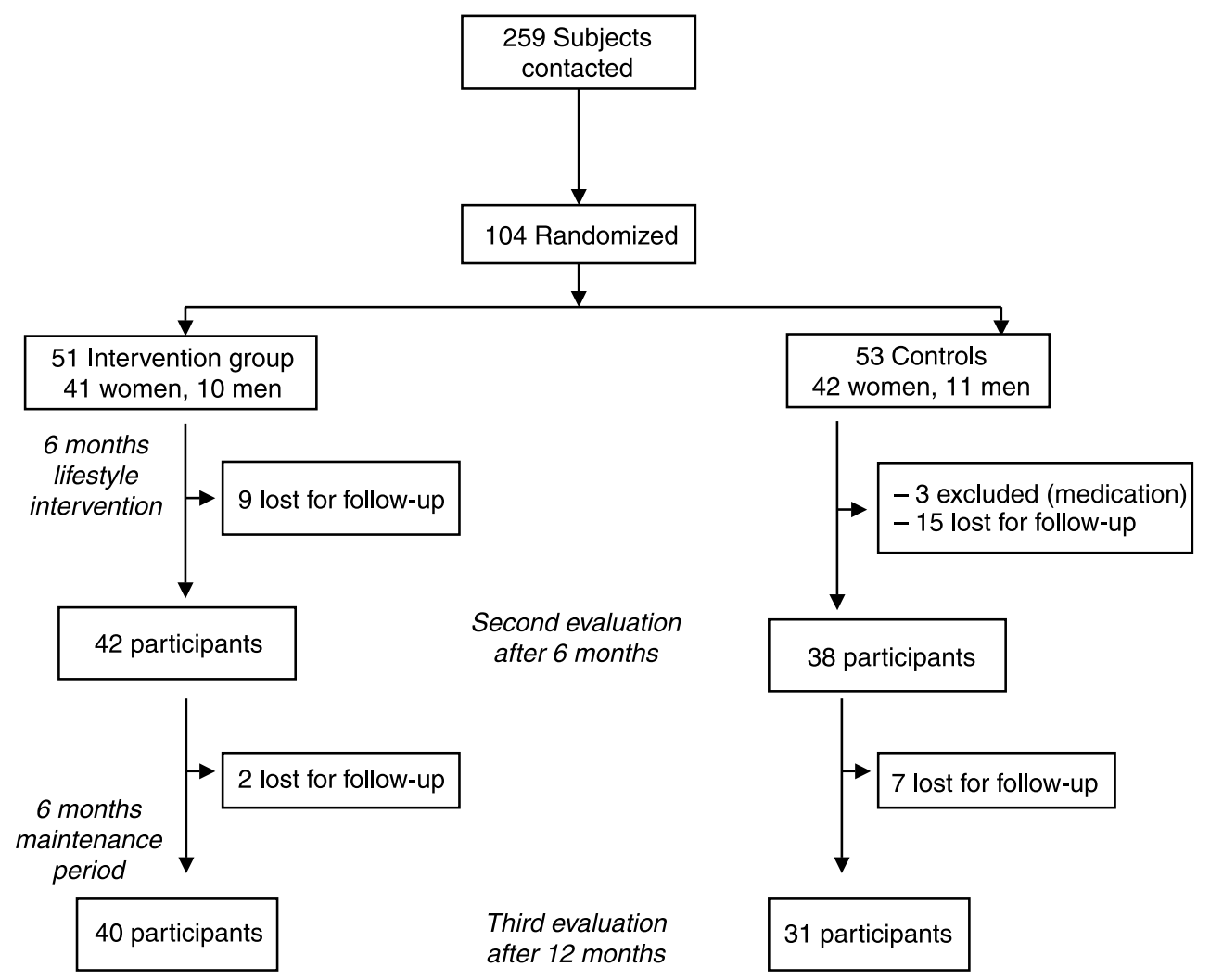

Fig. 1 Flow chart of the randomised controlled intervention trial 
Table 1 Baseline values of variables for the intervention and control groups

\begin{tabular}{|c|c|c|}
\hline & Intervention & Control \\
\hline$n$ & 51 & 53 \\
\hline Age (years) & $45 \pm 8$ & $46 \pm 10$ \\
\hline Weight (kg) & $75.4 \pm 10.3$ & $72.5 \pm 8.6$ \\
\hline BMI $\left(\mathrm{kg} \mathrm{m}^{-2}\right)$ & $28.8 \pm 2.4$ & $28.7 \pm 2.6$ \\
\hline Waist circumference $(\mathrm{cm})$ & $89.9 \pm 8.6$ & $89.3 \pm 7.9$ \\
\hline Fasting cholesterol $\left(\mathrm{mmoll}^{-1}\right)$ & $5.45 \pm 1.38$ & $5.24 \pm 1.0$ \\
\hline Fasting LDL cholesterol $\left(\mathrm{mmol}^{-1}\right)$ & $3.68 \pm 1.19$ & $3.46 \pm 0.94$ \\
\hline Fasting HDL cholesterol (mmoll $\left.{ }^{-1}\right)$ & $1.27 \pm 0.39$ & $1.22 \pm 0.36$ \\
\hline Fasting triglycerides $\left(\mathrm{mmoll}^{-1}\right)$ & $1.43 \pm 0.75$ & $1.71 \pm 10.05$ \\
\hline Fasting glucose $\left(\mathrm{mmoll}^{-1}\right)$ & $5.18 \pm 0.52$ & $5.15 \pm 0.52$ \\
\hline $2 \mathrm{~h}$ glucose $\left(\mathrm{mmoll}^{-1}\right)$ & $6.03 \pm 1.44$ & $5.98 \pm 1.50$ \\
\hline \multicolumn{3}{|l|}{ Blood pressure } \\
\hline Systolic (mmHg) & $115.1 \pm 14.2$ & $118.1 \pm 20.4$ \\
\hline Diastolic $(\mathrm{mmHg})$ & $77.6 \pm 23.3$ & $77.5 \pm 11.7$ \\
\hline \multicolumn{3}{|c|}{ Daily energy and selected nutrient intakes } \\
\hline Energy (kcal) & $2580 \pm 863$ & $2543 \pm 945$ \\
\hline Cholesterol (mg) & $316 \pm 155$ & $327 \pm 172$ \\
\hline Total fibre $(\mathrm{g})$ & $16.2 \pm 7.5$ & $13.2 \pm 6.7$ \\
\hline \multicolumn{3}{|l|}{$\%$ Energy as } \\
\hline Total fat & $33.4 \pm 6.3$ & $32.4 \pm 6.3$ \\
\hline Saturated fat & $10.0 \pm 2.8$ & $9.7 \pm 2.7$ \\
\hline Protein & $15.4 \pm 3.8$ & $16.3 \pm 3.9$ \\
\hline Carbohydrates & $50.2 \pm 8.9$ & $49.8 \pm 8.5$ \\
\hline \multicolumn{3}{|l|}{ Physical activity } \\
\hline Walking (min week ${ }^{-1}$ ) & $60(0.0,180)$ & $105(0.0,360)$ \\
\hline
\end{tabular}

BMI - body mass index; LDL - low-density lipoprotein; HDL - high-density lipoprotein.

Data are reported as mean \pm standard deviation or as median (interquartile range).

were no differences between groups in glucose tolerance status. The decrease in consumption of total energy, percentage of energy from fat, saturated fat and dietary cholesterol was greater in the intervention than in the control group at the 12-month follow-up $(P<0.04)$. The consumption of total fibre in the intervention group was significantly greater than in the control group $(P=0.01$; data not shown).

The goals of the dietary intervention were more often reached by participants from the intervention group (Table 3). At 6- and 12-month follow-up, the proportion of subjects in the intervention group who increased their intake of fruits (at least 2 portions day ${ }^{-1}$ ), use of olive oil and reduced their intake of saturated fat $(<10 \%$ of energy) was significantly higher than in the control group. For the vegetable intake goal (at least 5 portions day ${ }^{-1}$ ) and the fibre goal ( $>15 \mathrm{~g}$ daily) the proportion of subjects in the intervention group who achieved success was significantly higher at the 6-month follow-up $(P<0.01)$. The proportion of individuals who succeeded in achieving the goal of walking (at least $30 \mathrm{~min} \mathrm{day}^{-1}$ ) did not differ between groups.

\section{Discussion}

The present study provides evidence that nutritional intervention programmes to reduce the risk of type 2 diabetes and related diseases among overweight subjects are feasible at primary health-care centres. A second finding was the significant weight loss and decreased waist circumference, total cholesterol and LDL cholesterol with only three individualised dietary sessions at the 6-month follow-up, suggesting that less intensive lifestyle programmes with reduced resources are effective in changing food consumption and improving metabolic control and quality of life.

One of the limitations of the present study was the short time of follow-up, which may have restricted the ability to detect meaningful long-term differences. Also, the study population came from only one health-care unit and the nutritional counselling was delivered by a single nutritionist, limiting the extrapolation of our results.

However, intervening in overweight Brazilian adults at a health-care unit can be a useful approach, because social, economic and cultural aspects may influence the outcomes of lifestyle change programmes. To our knowledge, no epidemiological research has been carried out thus far to prevent type 2 diabetes in Brazil or other Latin American countries.

There are some reasons to believe that nutritional counselling is effective. First, the data were analysed following the intention-to-treat principle, even though some subjects in the dietary intervention group did not follow the recommendations about healthy lifestyle. Second, at baseline, all participants received written information and a 30 min group session addressing the importance of a healthy lifestyle and the control group may have benefited from that.

In the present study, the mean weight reduction (\% of baseline data) during the lifestyle intervention period at 6-month follow-up was modest $(-3.1 \%)$ and lower than that observed in more intensive lifestyle programmes, such as DPP $(-5.9 \%)$, DPS $(-5.1 \%)$ and the study conducted by Wing et al. ${ }^{10}$ ( $-5.5 \%$ in the diet group). On the other hand, the improvement in total cholesterol $(-12.3 \%)$ in our study after the intervention period was greater than that observed in the DPS $(-2.3 \%)$, in the prevention study of Wing et al. ${ }^{10}(-6.7 \%$ in the diet plus exercise group) and in most of the randomised clinical trials with nutritional counselling to lower blood cholesterol in free-living subjects, in which the mean reduction was around $5.5 \%$ at 12 months of intervention ${ }^{11}$.

The same tendency was observed in the reduction of LDL cholesterol in our study $(-15.5 \%)$ when compared with the study of Wing et al. ${ }^{10}(-9.8 \%$ in the diet group and $-5.0 \%$ in the diet and exercise group) at 6-month follow-up. The highly successful improvement in lipid profile may be attributed to the dietary changes in the intervention group. Besides the reduction in total fat and dietary cholesterol and the increase in the consumption of fruits and vegetables, more than $90 \%$ of the population reported the use of olive oil as salad dressing and the consumption of less than 10\% of energy from saturated fat. The proportion of individuals who achieved this goal was 
Table 2 Changes in selected clinical and metabolic variables from baseline to 6- and 12-month follow-up

\begin{tabular}{|c|c|c|c|c|c|}
\hline & \multicolumn{2}{|c|}{ Intervention } & \multicolumn{2}{|c|}{ Control } & \multirow[b]{2}{*}{$P$-value ${ }^{*}$} \\
\hline & Mean \pm SD & $95 \% \mathrm{Cl}$ & Mean \pm SD & $95 \% \mathrm{Cl}$ & \\
\hline \multicolumn{6}{|l|}{ Weight (kg) } \\
\hline 6-month & $-2.4 \pm 2.5$ & $-3.2,-1.6$ & $-0.3 \pm 2.3$ & $-1.1,0.4$ & $<0.001$ \\
\hline 12-month & $-2.3 \pm 3.3$ & $-3.4,-1.3$ & $-0.3 \pm 2.9$ & $-1.4,0.8$ & $<0.001$ \\
\hline \multicolumn{6}{|c|}{ Change in weight (\%) } \\
\hline 6-month & $-3.1 \pm 3.1$ & $-4.1,-2.1$ & $-0.4 \pm 3.2$ & $-1.4,0.64$ & $<0.001$ \\
\hline 12-month & $-3.4 \pm 4.6$ & $-4.8,-1.9$ & $-0.6 \pm 4.1$ & $-2.1,0.87$ & 0.012 \\
\hline \multicolumn{6}{|l|}{$\mathrm{BMI}\left(\mathrm{kg} \mathrm{m}^{-2}\right)$} \\
\hline 6 -month & $-0.8 \pm 0.9$ & $-1.1,-0.5$ & $-0.1 \pm 1.1$ & $-0.5,0.2$ & 0.06 \\
\hline 12-month & $-0.9 \pm 1.3$ & $-1.3,-0.5$ & $-0.2 \pm 1.3$ & $-0.6,0.3$ & 0.02 \\
\hline \multicolumn{6}{|c|}{ Waist circumference $(\mathrm{cm})$} \\
\hline 6-month & $-1.9 \pm 4.0$ & $-3.2,-0.7$ & $0.2 \pm 3.6$ & $-0.9,1.4$ & 0.01 \\
\hline 12-month & $-1.9 \pm 3.7$ & $-3.2,-0.8$ & $0.1 \pm 3.3$ & $-1.1,1.3$ & 0.01 \\
\hline \multicolumn{6}{|c|}{ Cholesterol $\left(\mathrm{mmoll}^{-1}\right)$} \\
\hline 6-month & $-0.67 \pm 1.3$ & $-1.1,-0.3$ & $-0.01 \pm 1.0$ & $-0.35,0.3$ & 0.01 \\
\hline 12-month & $-0.52 \pm 1.2$ & $-0.9,-0.1$ & $-0.28 \pm 0.6$ & $-0.53,-0.04$ & 0.33 \\
\hline \multicolumn{6}{|c|}{ LDL cholesterol $\left(\mathrm{mmoll}^{-1}\right)$} \\
\hline 6-month & $-0.57 \pm 1.2$ & $-0.9,-0.2$ & $0.14 \pm 1.0$ & $-0.2,0.4$ & 0.005 \\
\hline 12-month & $-0.49 \pm 0.9$ & $-0.8,-0.2$ & $-0.11 \pm 0.6$ & $-0.3,0.1$ & 0.06 \\
\hline \multicolumn{6}{|c|}{$\mathrm{HDL}$ cholesterol $\left(\mathrm{mmoll}^{-1}\right)$} \\
\hline 6-month & $0.005 \pm 0.24$ & $-0.06,0.08$ & $0.01 \pm 0.2$ & $-0.05,0.07$ & 0.95 \\
\hline 12-month & $-0.007 \pm 0.21$ & $-0.07,0.06$ & $0.00 \pm 0.2$ & $-0.08,0.08$ & 0.88 \\
\hline \multicolumn{6}{|c|}{ Fasting triglycerides $\left(\mathrm{mmoll}^{-1}\right)$} \\
\hline 6-month & $-0.1 \pm 0.6$ & $-0.3,0.1$ & $-0.07 \pm 0.8$ & $-0.34,0.19$ & 0.87 \\
\hline 12-month & $-0.05 \pm 0.7$ & $-0.25,0.24$ & $-0.18 \pm 0.8$ & $-0.47,0.12$ & 0.36 \\
\hline \multicolumn{6}{|c|}{ Fasting plasma glucose $\left(\mathrm{mmoll}^{-1}\right)$} \\
\hline 6-month & $-0.29 \pm 0.66$ & $-0.49,-0.08$ & $0.01 \pm 0.64$ & $-0.20,0.22$ & 0.04 \\
\hline 12-month & $0.005 \pm 0.73$ & $-0.22,0.24$ & $0.08 \pm 0.51$ & $-0.11,0.26$ & 0.63 \\
\hline \multicolumn{6}{|c|}{$2 \mathrm{~h}$ glucose $\left(\mathrm{mmoll}^{-1}\right)$} \\
\hline 6-month & $-0.97 \pm 1.34$ & $-1.39,-0.54$ & $-0.63 \pm 1.24$ & $-1.04,-0.21$ & 0.25 \\
\hline 12-month & $0.32 \pm 1.39$ & $-0.13,0.78$ & $0.65 \pm 1.42$ & $0.28,1.34$ & 0.15 \\
\hline \multicolumn{6}{|c|}{ Blood pressure } \\
\hline \multicolumn{6}{|c|}{ Systolic $(\mathrm{mmHg})$} \\
\hline 6-month & $1.8 \pm 14.1$ & $-2.5,6.2$ & $5.3 \pm 18.1$ & $-0.6,11.3$ & 0.33 \\
\hline 12-month & $-0.8 \pm 12.3$ & $-4.7,3.1$ & $3.1 \pm 13.0$ & $-1.7,7.9$ & 0.20 \\
\hline \multicolumn{6}{|c|}{ Diastolic (mmHg) } \\
\hline 6-month & $-1.3 \pm 8.9$ & $-4.1,1.4$ & $3.3 \pm 11.6$ & $-0.5,7.1$ & 0.049 \\
\hline 12-month & $-1.3 \pm 8.9$ & $-4.1,1.6$ & $3.5 \pm 7.4$ & $0.8,6.2$ & 0.02 \\
\hline
\end{tabular}

SD - standard deviation; BMI - body mass index; LDL - low-density lipoprotein; HDL - high-density lipoprotein.

${ }^{*} P$-values for the difference between the groups were determined by the $t$-test.

much higher than that observed in the Finnish DPS at 12-month follow-up (26\% of subjects in the intervention group $)^{12}$. This improvement in lipid profile could be responsible for a reduction of about $30-50 \%$ in the risk of cardiovascular diseases ${ }^{13}$ and improvement in endothelial function rather than moderate weight loss ${ }^{14}$.

The goal for fibre intake in the Finnish DPS was $15 \mathrm{~g} / 1000 \mathrm{kcal}$ daily and $25 \%$ of the intervention group accomplished this consumption. We believe that the main source of fibre for the Finnish population comes from highfibre cereals - an expensive approach for developing countries. In our study, the intervention group was encouraged to increase the intake of fibre (at least $15 \mathrm{~g} \mathrm{day}^{-1}$ ) by a higher consumption of foods naturally rich in fibre (such as fruits and vegetables), and about 50\% of the subjects reported achieving this goal.

Current evidence points to the beneficial effects of eating at least five daily portions of fruits and vegetables on the prevention of cancer and cardiovascular disease.
A randomised clinical trial conducted in the UK investigated the effect of intervention for increasing fruit and vegetable consumption in the health-care system on plasma concentration of antioxidant vitamins, daily fruit and vegetable intake, and blood pressure ${ }^{15}$. At 6-month follow-up, the self-reported fruit and vegetable intake increased by a mean (standard deviation) of 1.4 (1.7) portions in the intervention group. In our study, the reported increase in fruits and vegetables was 2.2 (3.1) portions, and $70 \%$ of the individuals in the intervention group reported eating the five portions daily proposed as a goal versus $18 \%$ in the control group at the 6-month follow-up ( $P<0.001$; data not shown).

Besides the non-significant difference between groups in the proportion of subjects achieving the physical activity goal, this proportion in the control group remained the same throughout the study. Among individuals in the intervention group, after 6 and 12 months of follow-up this proportion was double that observed at baseline. Mean 
Table 3 Success in achieving the intervention goals by the two groups at baseline, 6- and 12-month follow-up

\begin{tabular}{|c|c|c|c|}
\hline & Intervention & Control & $P$-value* \\
\hline \multicolumn{4}{|c|}{ Saturated fat ( $<10 \%$ of energy) } \\
\hline Baseline & 47.6 & 52.9 & 0.35 \\
\hline 6-month & 90.5 & 44.7 & $<0.001$ \\
\hline 12-month & 83.3 & 36.8 & $<0.001$ \\
\hline \multicolumn{4}{|c|}{ Fruits (at least 2 portions day ${ }^{-1}$ ) } \\
\hline Baseline & 28.6 & 18.4 & 0.28 \\
\hline 6-month & 47.6 & 23.7 & 0.02 \\
\hline 12-month & 50.0 & 20.0 & 0.01 \\
\hline \multicolumn{4}{|c|}{ Vegetables (at least 5 portions day ${ }^{-1}$ ) } \\
\hline Baseline & 23.8 & 7.9 & 0.054 \\
\hline 6-month & 42.9 & 7.9 & $<0.001$ \\
\hline 12-month & 36.1 & 20.0 & 0.17 \\
\hline \multicolumn{4}{|c|}{ Use of olive oil as salad dressing } \\
\hline Baseline & 54.9 & 52.8 & 0.83 \\
\hline 6-month & 92.9 & 44.7 & $<0.001$ \\
\hline 12-month & 95.0 & 61.3 & $<0.001$ \\
\hline \multicolumn{4}{|c|}{ Fibre (at least $15 \mathrm{~g} \mathrm{day}^{-1}$ ) } \\
\hline Baseline & 50.0 & 31.6 & 0.09 \\
\hline 6-month & 59.5 & 28.9 & 0.006 \\
\hline 12-month & 55.6 & 32.0 & 0.06 \\
\hline \multicolumn{4}{|c|}{ Walking (at least 30 min day $^{-1}$ ) } \\
\hline Baseline & 23.8 & 23.7 & 0.99 \\
\hline 6-month & 42.9 & 34.2 & 0.42 \\
\hline 12-month & 41.7 & 24.0 & 0.15 \\
\hline
\end{tabular}

Data are reported as $\%$ of subjects achieving the goal.

${ }^{*} P$-values for the difference between groups were determined by the chi-square test.

walking time for the intervention group was 25 min day $^{-1}$, which is enough to reduce the risk to develop cardiovascular diseases ${ }^{16}$.

In conclusion, the present study provides evidence that diabetes prevention programmes are effective in changing lifestyle and risk factors for type 2 diabetes in overweight free-living individuals and can be implemented in healthcare systems with lower resources.

\section{Acknowledgements}

We are grateful to Professor José Antônio Cordeiro for statistical contribution, and Raquel de Castro Monteiro and Patrícia Frizzas for valuable help in fieldwork. D.S.S. and E.C.S. had scholarships from FAPESP and CNPq, respectively. The work was supported by Fundação de Amparo a Pesquisa do Estado de São Paulo (FAPESP - 2000/01767-3) and Conselho Nacional de Desenvolvimento Científico e Tecnológico (CNPq).

\section{References}

1 King H, Aubert RE, Herman WH. Global burden of diabetes, 1995-2025. Diabetes Care 1998; 21: 1414-31.

2 World Health Organization (WHO)/Food and Agriculture Organization (FAO). Diet, Nutrition and the Prevention of
Chronic Diseases. Joint WHO/FAO Expert Consultation. Geneva: WHO/FAO, 2003.

3 Diabetes Prevention Program Group. Reduction in the incidence of type 2 diabetes with lifestyle intervention or metformin. New England Journal of Medicine 2002; 346: 393-403.

4 Tuomilehto J, Lindstrom J, Eriksson JG, Valle TT, Hamalainen $\mathrm{H}$, Iianne-Parika $\mathrm{P}$, et al. for The Finnish Diabetes Prevention Study Group. Prevention of type 2 diabetes mellitus by changes in lifestyle among subjects with impaired glucose tolerance. New England Journal of Medicine 2001; 344: 1343-50.

5 American Diabetes Association and National Institute of Diabetes, Digestive and Kidney Diseases. The prevention or delay of type 2 diabetes. Diabetes Care 2002; 25: 742-9.

6 Alberti KGMM, Zimmet PZ. for the WHO Consultation. Definition, diagnosis and classification of diabetes mellitus and its complications. Part 1: diagnosis and classification of diabetes mellitus. Provisional report of a WHO consultationDiabetic Medicine 1998; 15: 539-53.

7 Cardoso MA, Kida AA, Tomita LY, Stocco PR. Reproducibility and relative validity of a food frequency questionnaire among women of Japanese ancestry living in Brazil. Nutrition Research 2001; 21: 725-33.

8 Ribeiro AB, Cardoso MA. Development of a food frequency questionnaire as a tool for programs of chronic diseases prevention. Revista de Nutricao 2002; 15: 201-7 (in Portuguese).

9 Block G, Coyle LM, Hartman AM, Scoppa SM. Revision of dietary analysis software for the health habits and history questionnaire. American Journal of Epidemiology 1994; 139: 1190-6.

10 Wing RR, Venditti E, Jakicic JM, Polley BA, Lang W. Lifestyle intervention in overweight individual with a family history of diabetes. Diabetes Care 1998; 21: 350-9.

11 Tang JL, Armitage JM, Lancaster T, Silagy CA, Fowler GH, Neil HAW. Systematic review of dietary intervention trials to lower blood total cholesterol in free-living subjects. British Medical Journal 1998; 316: 1213-20.

12 Lindstrom J, Louheranta A, Mannelin M, Rastas M, Salminen V, Eriksson J, et al. for the Finnish Diabetes Prevention Study Group. Lifestyle intervention and 3-year results on diet and physical activity. Diabetes Care 2003; 26 : 3230-6.

13 Law MR, Wald NJ, Thompson SG. By how much and how quickly does reduction in serum cholesterol concentration lower risk of ischaemic heart disease? British Medical Journal 1994; 308: 367-72.

14 Bergholm R, Tiikkainen M, Vehkavara S, Tamminen M, Teramo K, Rissanen A, et al. Lowering of LDL cholesterol rather than moderate weight loss improves endotheliumdependent vasodilatation in obese women with previous gestational diabetes. Diabetes Care 2003; 26: 1667-72.

15 John JH, Ziebland S, Yudkin P, Roe LS. Neil HAW for the Oxford Fruit and Vegetable Study Group. Effects of fruit and vegetable consumption on plasma antioxidant concentrations and blood pressure: a randomized controlled trial. Lancet 2002; 359: 1969-74.

16 Manson JE, Hu FB, Rich-Edwards JW, Colditz GA, Stampfer MJ, Willett WC, et al. A prospective study of walking as compared with vigorous exercise in the prevention of coronary heart disease in women. New England Journal of Medicine 1999; 341: 650-8. 